\title{
Deformation Quantization by Moyal Star-Product and Stratonovich Chaos
}

\author{
Rémi LÉANDRE ${ }^{\dagger}$ and Maurice OBAME NGUEMA $\ddagger$ \\ † Laboratoire de Mathématiques, Université de Franche-Comté, 25030, Besançon, France \\ E-mail: remi.leandre@univ-fcomte.fr \\ ¥ Institut de Mathématiques de Bourgogne, Université de Bourgogne, 21000, Dijon, France \\ E-mail: maurice-saint-clair.obame-nguema@u-bourgogne.fr
}

Received November 16, 2011, in final form March 06, 2012; Published online March 15, 2012 http://dx.doi.org/10.3842/SIGMA.2012.011

\begin{abstract}
We make a deformation quantization by Moyal star-product on a space of functions endowed with the normalized Wick product and where Stratonovich chaos are well defined.
\end{abstract}

Key words: Moyal product; Connes algebra; Stratonovich chaos; white noise analysis

2010 Mathematics Subject Classification: 60H40; 81S20

\section{Introduction}

This work follows those of G. Dito [3] (motivated by quantum field theory [4, 5, 8, 27]), of R. Léandre [14, 15, 16] and G. Dito, R. Léandre [6]. [3] deals with the deformation quantization of Moyal on a Hilbert space: the condition of equivalence of the Moyal deformations is that the chosen perturbation operator is a Hilbert-Schmidt operator but in this case, Moyal and normal products are not equivalents. [15] choose Hida weighted Fock spaces which are very small spaces. This gives spaces of continuous functionals on the path space. The inequivalences of [3] become equivalences. In [6], the Malliavin test algebra is used for the Moyal quantization. A very important remark in [3] and [6] is that the matrix of the associated Poisson structure is still bounded.

Our motivation comes from the fact we deal with deformation quantization on an algebra constituted of Stratonovich chaos. The Connes spaces where our work was possible present some differences with the Hida spaces of [15]. Indeed, Connes spaces are involved with tensor products of Banach spaces and Hida spaces are involved with tensor products of Hilbert spaces [11, 17, 23]. In infinite dimension analysis, there are two basic objects [21]:

- an algebraic model;

- a mapping space and a map $\Psi$ defined from algebraic model into the space of functionals on this mapping space.

Let us recall what is the main difference between the point of view of [6] and the point of view of $[15,16]$ and this paper:

- The tools of Malliavin calculus on the Wiener space are used in [6]. The Malliavin test algebra is constituted of functionals almost surely defined, because there is no Sobolev imbedding in infinite dimension [19, 20, 22, 25].

- The tools of white noise analysis are used in $[15,16]$ and in this present work. The differential operations and the topological structures are seen at the level of the algebraic model and after they are transported through the map $\Psi$ on a set of functionals which 
are continuous on the Wiener space. [15] and [16] work on the level of the algebraic model. They use a different normalization of annihilation operators which fit with the map Stratonovitch chaos and not with the map Wiener chaos. In the present paper, we transport the algebraic operations of $[15,16]$ on a functional space by using the map $\Psi$ Stratonovitch chaos which was not defined in [15] and [16].

Note that E. Getzler [10] was the first to consider another map than the map Wiener chaos. He used as algebraic space a Connes space and as map $\Psi$ the map of Chen iterated integrals (see [13] for developments).

After this quick presentation, we shall define in the second part our Connes functional space on which the multiple Stratonovich integrals or Stratonovich chaos are well defined. We shall finish this second part by a study of the annihilation operator on our functions space. In the third part, the deformation quatization of the Poisson bracket by a Moyal product is defined on the Connes space. The last part is about equivalences of deformations on the Connes space.

\section{Gaussian space}

In this section we show the existence of a Gaussian measure on a space of continuous loops which will be our reference measure throughout this work on the algebraic space. We also define Stratonovich chaos and differentiation operators we use in the next section.

\subsection{Gaussian measure}

Let us consider the Hilbert space $\mathcal{H}:=\mathcal{H}\left(S^{1}, \mathbb{R}^{d}\right)$ such that $\gamma \in \mathcal{H}$ verifies: $\|\gamma\|^{2}=\int_{0}^{1}|\gamma(s)|^{2} d s+$ $\int_{0}^{1}|\dot{\gamma}(s)|^{2} d s$. We consider $B=\left\{B(t)=\left(B_{i}(t)\right), t \in S^{1}\right\}$ the Wiener process associated to this Hilbert space. We note $(\cdot, t) \mapsto G(\cdot, t)$ the symmetric Green kernel. Let $h$ be a continuous function from $S^{1}$ with values in $\mathbb{R}$ such that

$$
h(1)=\int_{0}^{1}\langle h(s), G(s, 1)\rangle d s+\int_{0}^{1}\left\langle\dot{h}(s), \frac{d}{d s} G(s, 1)\right\rangle d s .
$$

$G$ is solution of a second-class linear differential equation and the Green kernel of that equation verifies $\frac{d^{2}}{d s^{2}} G(s, 1)-G(s, 1)=0$ but also $\frac{d}{d s} G(1,1)-\frac{d}{d s} G(0,1)=1$. We obtain that $G(s, 1)=$ $\alpha e^{-s}+\beta e^{s}$ where $\alpha$ and $\beta$ are constants of integration with respect to $s$. With the conditions in the limits, we find that $\alpha=\frac{-1}{2\left(1-e^{-1}\right)}$ and $\beta=\frac{1}{2(1-e)}$. Moreover, we know that $E\left[B_{i}(s) B_{j}(t)\right]=$ $\delta_{i, j} G(s, t)$ where $\delta_{i, j}$ denotes the classical Kronecker symbol. There is $\mu>0$ such that $\mid G(t, t)+$ $G(s, s)-2 G(s, t)|\leq \mu| t-s \mid$. By standard result on Gaussian measures, for all $p>1$, there exist $\mu_{p}>0$ such that

$$
E\left[|B(t)-B(s)|^{2 p}\right] \leq \mu_{p}|t-s|^{p}
$$

By the Kolmogorov's criterion of continuity, we deduct that $B$ is Hoelderian.

\subsection{Connes space}

Let us consider the Hilbert space $\mathcal{H}$ above and a map $\gamma$ defined from the circle into $\mathbb{R}^{d}$ such that

$$
\int_{0}^{1}|\gamma(s)|^{2} d s+\int_{0}^{1}|\dot{\gamma}(s)|^{2} d s=\|\gamma\|^{2} .
$$


We consider a symmetric tensor product $F^{n}$

$$
F^{n}=\frac{1}{n !} \sum_{\sigma \in \mathfrak{G}_{n}} F_{\sigma(1)} \otimes \cdots \otimes F_{\sigma(n)},
$$

where $\sigma$ is a permutation of the symmetric group of degree $n . F_{i}$ are elements of $\mathcal{H}$ and $\otimes$ denotes the standard tensor product on this Hilbert space.

$F^{n}$ can be seen as a function from $\left(S^{1}\right)^{n}$ into $\left(\mathbb{R}^{d}\right)^{\otimes n}$. We consider the symmetric Fock space constituted of $F=\sum F^{n}$

$$
\sum\left(\left\|F^{n}\right\|^{\hat{\otimes} n}\right)^{2}<\infty
$$

where we consider the Hilbert norm on the symmetric tensor product $\mathcal{H}^{\hat{\otimes} n}$. We can define the Wiener chaos

$$
\left\langle F^{n}, B(\cdot) \otimes \cdots \otimes B(\cdot)\right\rangle_{W}
$$

It is well defined for the symmetric function $F^{n} \in \mathcal{H}^{\widehat{\otimes} n}$ and the Gaussian process $B$. The map Wiener chaos realizes as classical an isomorphism between the symmetric Fock space and the $L^{2}$ of the Wiener space. The goal of this work is to replace Wiener chaos by Stratonovitch chaos.

Let $\left\{e_{i}\right\}_{1 \leq i \leq d}$ be a canonical basis of $\mathbb{R}^{d}$. We get by Fourier expansion an orthonormal basis of the Hilbert space for some $\lambda>0$

$$
\gamma_{i, k}(s)=\frac{\cos (2 k \pi s)}{\sqrt{\lambda k^{2}+1}} e_{i}
$$

if $k \geq 0$ and if $k<0$

$$
\gamma_{i, k}(s)=\frac{\sin (2 k \pi s)}{\sqrt{\lambda k^{2}+1}} e_{i}
$$

Consider that $\left(\gamma_{i}\right)_{i \geq 1}$ is an orthonormal basis of $\mathcal{H}$, then an orthonormal basis of $\mathcal{H}^{\widehat{\otimes} n}$ takes the form

$$
\gamma_{N}(s)=\frac{1}{N !} \sum_{\sigma \in \mathfrak{S}_{N}} \gamma_{\sigma\left(j_{1}\right)}\left(s_{\sigma(1)}\right) \otimes \cdots \otimes \gamma_{\sigma\left(j_{N}\right)}\left(s_{\sigma(N)}\right) .
$$

$\otimes$ denotes the tensor product on $\mathbb{R}^{d} . \sigma$ is a permutation of the symmetric group $\mathfrak{S}_{N}$ of degree $N$. For all $l=1, \ldots, N$

$$
j_{l} \in \mathfrak{J}=\bigcup_{p=1}^{m} \mathfrak{J}_{n_{p}}
$$

and $\mathfrak{J}_{n_{p}}=\left\{\left(i_{p}, k_{p}\right), \ldots,\left(i_{p}, k_{p}\right)\right\}$ such that $\left|\mathfrak{J}_{n_{p}}\right|=n_{p}$ and $\mathfrak{J}_{n_{i}} \cap \mathfrak{J}_{n_{j}}=\varnothing$ if $i \neq j$ and we note by $|\mathfrak{J}|=N=\sum_{p=1}^{m} n_{p}$ for $n_{p} \leq n$.

Remark 1. We shall use the orthonormal basis of the symmetric space $\mathcal{H}^{\widehat{\otimes} n}$ given by (5) to avoid redundancies in the calculations throughout this work.

Let us consider the space $\mathrm{CO}_{k, C}$ of function $F$ given by

$$
\sum F^{n}=F,
$$


where $F^{n} \in \mathcal{H}^{\widehat{\otimes} n}$ is a $C^{\infty}$ function of $n$ parameters such that for all $k, C>0$

$$
\|F\|_{k, C}=\sum C^{n} \sum_{\substack{J_{\alpha} \cap J_{\beta}=\varnothing \\ J_{1} \cup \cdots \cup J_{l}=\{1, \ldots, n\}}}\left\|D_{S_{J_{1}}}^{\left(n_{1}\right)} \cdots D_{S_{J_{l}}}^{\left(n_{l}\right)} F^{n}\right\|_{\infty}:=\sum C^{n}\left\|F^{n}\right\|_{k}<\infty,
$$

where $J_{\alpha}=\left\{l_{1}, \ldots, l_{\alpha}\right\}$ with $J_{1} \cup \cdots \cup J_{l}=\{1, \ldots, n\}$ and $S_{J_{\alpha}}=\left\{s_{l_{1}}, \ldots, s_{l_{\alpha}}\right\}$. For $\alpha \in\{1, \ldots, l\}$ such that $n_{\alpha} \leq k$, we write

$$
D_{S_{J_{\alpha}}}^{\left(n_{\alpha}\right)}=\frac{\partial^{\sum n_{\alpha}}}{\partial s_{l_{1}}^{n_{\alpha}} \cdots \partial s_{l_{\alpha}}^{n_{\alpha}}}
$$

Definition 1. We call Connes space the set $\mathrm{CO}_{\infty}$ - given by

$$
\mathrm{CO}_{\infty-}=\bigcap_{k, C} \mathrm{CO}_{k, C}
$$

for all $k, C>0$.

Remark 2. A sequence $F_{k}$ of $\mathrm{CO}_{\infty_{-}}$converges to $F$ in $\mathrm{CO}_{\infty_{-}}$if it converges in all $\mathrm{CO}_{k, C}$.

Remark 3. Let us compare with the Hida Fock space. We consider the positive selfadjoint Laplacian $\Delta$ on $\mathcal{H}$ and we consider the $k^{\text {th }}$ order Sobolev space $\mathcal{H}_{k}$ associated with $\Delta+1$. It is a Hilbert space. We consider the symmetric tensor product $\mathcal{H}_{k}^{\widehat{\otimes} n}$ endowed with its Hilbert norm $\|\cdot\|_{k}^{\hat{n}}$. Let us consider a sequence $F^{n}$ of $\mathcal{H}_{k}^{\widehat{\otimes} n} . \quad F=\sum F^{n}$ belongs to $W . N_{k, C}$ if $\sum C^{n}\left(\|F\|_{k}^{\hat{\otimes} n}\right)^{2}$ is finite. The Hida Fock space $W \cdot N_{\infty-}$ is the intersection of all $W . N_{k, C}$. By Cauchy-Schwarz inequality and the Sobolev imbedding theorem we see that the Connes space is densely continuously imbedded in the Hida Fock space if we consider the standard Hilbert norm $\|\cdot\|_{k}^{\otimes n}$ on the symmetric tensor product of $\mathcal{H}$ in the definition of the Hida Fock space.

Definition 2. Let $F=\sum F^{n}$ and $G=\sum G^{m}$ be two functions of $\mathrm{CO}_{\infty-}$. We define Wick product of $F$ and $G$ by

$$
: F \cdot G:=\sum_{m, n} F^{n} \widehat{\otimes} G^{m} .
$$

$F^{n} \widehat{\otimes} G^{m}$ is the symmetric tensor product of the functions $F^{n}$ and $G^{m}$ given by

$$
F^{n} \widehat{\otimes} G^{m}=\frac{1}{(m+n) !} \sum_{\sigma \in \mathfrak{S}_{n+m}} F^{n} \otimes_{\sigma} G^{m}
$$

with $F^{n}$ and $G^{m}$ verifying both (1) and (2) and where $\sigma$ is a permutation of the symmetric group $\mathfrak{S}_{n+m}$ of the space $\mathcal{H}^{\otimes(m+n)}$.

Theorem 1. The Connes space $\mathrm{CO}_{\infty-}$ is a topological commutative algebra for the Wick product.

Proof. For the algebraic properties of the Wick product on $\mathrm{CO}_{\infty-}$, see Theorem 2 and Proposition 2. Let us consider

$$
F=\sum F^{n}, \quad G=\sum G^{m} .
$$

By (6) and (7) we have

$$
: F . G:=\sum_{m, n} \frac{1}{(m+n) !} \sum_{\sigma \in\{1, \ldots, n+m\}} F^{n} \otimes_{\sigma} G^{m} .
$$


Clearly, for all $k>0$, there exist $k_{0}>0$ such that

$$
\left\|F^{n} \otimes_{\sigma} G^{m}\right\|_{k} \leq\left\|F^{n}\right\|_{k_{0}}\left\|G^{m}\right\|_{k_{0}}
$$

For $C>0$, there exist $C_{0}>0$ such that

$$
\|: F . G:\|_{k, C} \leq \sum_{n, m} C_{0}^{m} C_{0}^{n}\left\|F^{n}\right\|_{k_{0}}\left\|G^{m}\right\|_{k_{0}} .
$$

We deduce

$$
\|: F . G:\|_{k, C} \leq K\|F\|_{k_{0}, C_{0}}\|G\|_{k_{0}, C_{0}} .
$$

The theorem is proved.

\subsection{Multiple Stratonovich integrals}

The theory of Stratonovich chaos was initiated in $[12,24]$ but no convenient functional space was defined. Let us consider $\gamma_{N}=\gamma_{i_{1}, k_{i_{1}}}^{\otimes n_{1}} \otimes \cdots \otimes \gamma_{i_{m}, k_{i_{m}}}^{\otimes n_{m}}$ such that $\gamma_{i_{j}, k_{i_{j}}}^{\otimes n_{j}}$ is the $n_{j}$-th tensor product of $\gamma_{i_{j}, k_{i}} \in H$. The associated Stratonovitch chaos takes the form

$$
\begin{aligned}
& I_{m}^{S}\left(\gamma_{N}\right)=\prod_{1 \leq j \leq m}\left\langle\gamma_{i_{j}, k_{i_{j}}}^{\otimes n_{j}}, B(\cdot) \otimes \cdots \otimes B(\cdot)\right\rangle_{S} \\
& I_{m}^{S}\left(\gamma_{N}\right)=\prod_{1 \leq j \leq m}\left\langle\gamma_{i_{j}, k_{i_{j}}}, B(\cdot)\right\rangle^{n_{j}}
\end{aligned}
$$

We consider multiple Stratonovitch integrals. In Stratonovitch calculus, the Itô formula reduces to the classical one. So (8) gives (9), because in such a case the Stratonovitch-Itô formula is nothing else than the ordinary Fubini theorem. We have

$$
I_{m}^{S}\left(\gamma_{N}\right)=\prod_{1 \leq j \leq m}\left(\int_{S^{1}}\left\langle\gamma_{i_{j}, k_{i_{j}}}\left(s_{j}\right), B\left(s_{j}\right)\right\rangle d s_{j}+\int_{S^{1}}\left\langle\dot{\gamma}_{i_{j}, k_{i_{j}}}\left(s_{j}\right), \circ d B\left(s_{j}\right)\right\rangle\right)^{n_{j}}
$$

where we consider a Stratonovitch differential $\circ d B\left(s_{j}\right)$. With the integration by parts formula

$$
\int_{S^{1}}\left\langle\dot{\gamma}\left(s_{j}\right), d B\left(s_{j}\right)\right\rangle=-\int_{S^{1}}\left\langle\frac{\partial^{2}}{\partial s_{j}^{2}} \gamma\left(s_{j}\right), B\left(s_{j}\right)\right\rangle d s_{j},
$$

we get

$$
I_{m}^{S}\left(\gamma_{N}\right)=\prod_{1 \leq j \leq m}\left(\int_{S^{1}}\left\langle\gamma_{i_{j}, k_{i}}\left(s_{j}\right), B\left(s_{j}\right)\right\rangle d s_{j}-\int_{S^{1}}\left\langle\frac{\partial^{2}}{\partial s_{j}^{2}} \gamma_{i_{j}, k_{i_{j}}}\left(s_{j}\right), B\left(s_{j}\right)\right\rangle d s_{j}\right)^{n_{j}} .
$$

Then

$$
\begin{aligned}
I_{m}^{S}\left(\gamma_{N}\right)= & \int_{\left(S^{1}\right)^{N}} \prod_{l_{1}=1}^{n_{1}} \cdots \prod_{l_{n_{m}}=n_{m-1}+1}^{n_{m}}\left(\left\langle\gamma_{i_{1}, k_{i_{1}}}\left(s_{l_{1}}\right), B\left(s_{l_{1}}\right)\right\rangle\right. \\
& \left.-\left\langle\frac{\partial^{2}}{\partial s_{l_{1}}^{2}} \gamma_{i_{1}, k_{i_{1}}}\left(s_{1_{1}}\right), B\left(s_{l_{1}}\right)\right\rangle\right) \cdots\left(\left\langle\gamma_{i_{n_{m}}, k_{i_{n_{m}}}}\left(s_{l_{n_{m}}}\right), B\left(s_{l_{n_{m}}}\right)\right\rangle\right. \\
& \left.-\left\langle\frac{\partial^{2}}{\partial s_{l_{n_{m}}}^{2}} \gamma_{i_{n_{m}}, k_{i_{m}}}\left(s_{l_{n_{m}}}\right), B\left(s_{l_{n_{m}}}\right)\right\rangle\right) d s_{l_{1}} \cdots d s_{N},
\end{aligned}
$$


and finally

$$
I_{m}^{S}\left(\gamma_{N}\right)=\sum_{J \subset\{1, \ldots, N\}} \int_{\left(S^{1}\right)^{N}}(-1)^{N-|J|} \prod_{\substack{j \in J \\ l \notin J}}\left\langle\gamma_{i_{j}, k_{i_{j}}}\left(s_{j}\right), B\left(s_{j}\right)\right\rangle\left\langle\frac{\partial^{2}}{\partial s_{l}^{2}} \gamma_{i_{l}, k_{i_{l}}}\left(s_{l}\right), B\left(s_{l}\right)\right\rangle d s_{j} d s_{l} .
$$

These considerations, which take care of the difference of behaviour between Stratonovitch chaos and Wiener chaos into the passage from (8) to (9) allow us to give the next definition and to generalize by linearity $I_{m}^{S}\left(\gamma_{N}\right)$ in the functions $F:=\sum F^{n}$ which are not products. We get

Definition 3. The multiple Stratonovich integrals or Stratonovich chaos takes the form

$$
I_{m}^{S}(F)=\sum_{n} \sum_{J \subset\{1, \ldots, n\}} \int_{\left(S^{1}\right)^{n}}(-1)^{n-|J|}\left\langle D_{S_{J}}^{(2)} F^{n}\left(s_{1}, \ldots, s_{n}\right), B\left(s_{1}\right) \otimes \cdots \otimes B\left(s_{n}\right)\right\rangle d s_{1} \cdots d s_{n},
$$

and we put

$$
I_{m}^{S}(F):=\sum_{n}\left\langle F^{n}, B \otimes \cdots \otimes B\right\rangle_{S} .
$$

Remark 4. For arbitrary small $\mu>0$, we can choose $n_{\mu}>0\left(n_{\mu}\right.$ depending only on $\left.\mu\right)$ such that for all $n>n_{\mu}$, we have $\sup _{s}\left|D_{S_{J}}^{(2)} F^{n}(s)\right| \leq \mu^{n}$ and for all $\mathbf{M}>0$ such that $\|B\|_{\infty}:=$ $\sup \left(\left\{|B(s)|, s \in S^{1}\right\}\right) \leq \mathbf{M}$, we get

$$
\sup \left\{\left|\left\langle D_{S_{J}}^{(2)} F^{n}\left(s_{1}, \ldots, s_{n}\right), B\left(s_{1}\right) \otimes \cdots \otimes B\left(s_{n}\right)\right\rangle\right| s_{1}, \ldots, s_{n} \in S^{1}\right\} \leq \mathbf{M}^{n} \mu^{n} .
$$

Then, there is $C_{\mu}>0$ such that

$$
\sum_{m \geq 1}\left\|I_{m}^{S}(F)\right\|_{\infty} \leq \sum_{n>n_{\mu}} 2^{n} \mu^{n} \mathbf{M}^{n}+C_{\mu}<\infty
$$

where we define $\left\|I_{m}^{S}(F)\right\|_{\infty}:=\sup \left(\left\{\left|I_{m}^{S}(F)\right|,\|B\|_{\infty} \leq \mathbf{M}\right\}\right)$. Then $I_{m}^{S}(F)$ converges normally. Clearly the map $B \mapsto I_{m}^{S}(F)$ is continuous.

Theorem 2. The multiple Stratonovich integrals of the Wick product of two functions of $\mathrm{CO}_{\infty-}$ is equal to the product of their multiple Stratonovich integrals for all $F, G \in \mathrm{CO}_{\infty-}$

$$
I_{m}^{S}(: F . G:)=I_{m}^{S}(F) \cdot I_{m}^{S}(G) .
$$

Proof. We consider $F=\sum F^{n_{1}}$ and $G=\sum G^{n_{2}}$ two functions of $\mathrm{CO}_{\infty_{-}}$. Since

$$
: F . G:=\frac{1}{\left(n_{1}+n_{2}\right) !} \sum_{\sigma \in \mathfrak{S}_{n_{1}+n_{2}}} F^{n_{1}} \otimes_{\sigma} G^{n_{2}},
$$

we have

$$
\begin{aligned}
I_{m}^{S}(: F . G:) & =\sum_{n_{1}, n_{2}} I_{m}^{S}\left(\frac{1}{\left(n_{1}+n_{2}\right) !} \sum_{\sigma \in \mathfrak{S}_{n_{1}+n_{2}}} F^{n_{1}} \otimes_{\sigma} G^{n_{2}}\right) \\
& =\sum_{n_{1}, n_{2}} \sum_{\sigma \in \mathfrak{S}_{n_{1}+n_{2}}} \frac{1}{\left(n_{1}+n_{2}\right) !}\left\langle F^{n_{1}} \otimes_{\sigma} G^{n_{2}}, B \otimes \cdots \otimes B\right\rangle_{S} .
\end{aligned}
$$

But, by permutating indexes, we have clearly

$$
\begin{aligned}
\left\langle F^{n_{1}} \otimes_{\sigma} G^{n_{2}}, B \otimes \cdots \otimes B\right\rangle= & \left\langle F^{n_{1}} \otimes G^{n_{2}}, B \otimes \cdots \otimes B\right\rangle_{S} \\
& \times\left\langle F^{n_{1}}, B \otimes \cdots \otimes B\right\rangle_{S}\left\langle G^{n_{2}}, B \otimes \cdots \otimes B\right\rangle_{S} .
\end{aligned}
$$


By using Fubini's theorem, we get

$$
\begin{aligned}
I_{m}^{S}(: F . G:)= & \sum_{n_{1}, n_{2}}\left\langle F^{n_{1}}, B \otimes \cdots \otimes B\right\rangle_{S}\left\langle G^{n_{2}}, B \otimes \cdots \otimes B\right\rangle_{S} \\
& =\left(\sum_{n_{1}}\left\langle F^{n_{1}}, B \otimes \cdots \otimes B\right\rangle_{S}\right)\left(\sum_{n_{2}}\left\langle G^{n_{2}}, B \otimes \cdots \otimes B\right\rangle_{S}\right)=I_{m}^{S}(F) \cdot I_{m}^{S}(G) .
\end{aligned}
$$

The theorem is proved.

\subsection{Differentiation operators}

Differentiation operators are annihilation and creation operators. In the case of Hida test algebra (7), these operators are adjoint operators and then their study is simplified. In our case, using Banach spaces to define the Connes space makes that it is difficult to give a definition of an adjoint operator. Then, we just give a description of annihilation operator.

Definition 4. We define annihilation operator on $\mathrm{CO}_{\infty_{-}}$, for all $h \in \mathcal{H}$ and $F=\sum F^{n} \in \mathrm{CO}_{\infty-}$ by

$$
a_{h} F:=\sum \int_{S^{1}}\left[\left\langle F^{n}\left(s_{1}, \ldots, \bar{s}_{i}, \ldots, s_{n-1}\right), h\left(\bar{s}_{i}\right)\right\rangle+\left\langle\frac{d}{d \bar{s}_{i}} F^{n}\left(s_{1}, \ldots, \bar{s}_{i}, \ldots, s_{n-1}\right), \dot{h}\left(\bar{s}_{i}\right)\right\rangle\right] d \bar{s}_{i},
$$

where $\bar{s}_{i}$ means that we make a concatenation at this term.

We have

Proposition 1. The Gâteaux derivative of a multiple Stratonovich integrals of a function $F \in$ $\mathrm{CO}_{\infty}$ - is equal to the multiple Stratonovich integral of the annihilation operator of that function. For all $F \in \mathrm{CO}_{\infty-}$

$$
D_{h} I_{m}^{S}(F)=I_{m}^{S}\left(a_{h} F\right) .
$$

Proof. We have

$$
\begin{gathered}
D_{h} I_{m}^{S}(F)=\sum_{n} \sum_{i} \sum_{J \subset\{1, \ldots, n\}} \int_{\left(S^{1}\right)^{n}}(-1)^{n-|J|} \sum\left\langle D_{S_{J}}^{(2)} F^{n}\left(s_{1}, \ldots, s_{i-1}, \bar{s}_{i}, s_{i+1}, \ldots, s_{n-1}\right),\right. \\
\left.B\left(s_{1}\right) \otimes \cdots \otimes h\left(\bar{s}_{i}\right) \otimes \cdots \otimes B\left(s_{n}\right)\right\rangle d s_{1} \cdots d s_{n} \\
=\sum_{n} \sum_{J \subset\{1, \ldots, n-1\}} \int_{\left(S^{1}\right)^{n-1}}(-1)^{n-|J|-1}\left\langle D_{S_{J}}^{(2)} a_{h} F^{n}\left(s_{1}, \ldots, s_{n-1}\right),\right. \\
\left.B\left(s_{1}\right) \otimes \cdots \otimes B\left(s_{n-1}\right)\right\rangle d s_{1} \cdots d s_{n-1} .
\end{gathered}
$$

The result holds.

Remark 5. Let be $h \in \mathcal{H}$. There is $C_{1}>C$ such that for for all $F=\sum F^{n} \in \mathrm{CO}_{\infty-}$

$$
\left\|a_{h} F\right\|_{k, C} \leq \sum C_{1}^{n}\|h\|\left\|F^{n+1}\right\|_{k}<\infty .
$$

The annihilation operator is continuous on $\mathrm{CO}_{\infty-}$.

Proposition 2. The annihilation operator is a derivation for the Wick product on $\mathrm{CO}_{\infty_{-}}$. For all $F, G \in \mathrm{CO}_{\infty-}$

$$
a_{h}(: F \cdot G:)=:\left(a_{h} F\right) \cdot G:+: F \cdot\left(a_{h} G\right): .
$$


Proof. We have just to show that the map $F \mapsto I_{m}^{S}(F)$ is injective. Indeed, considering (10) and the fact that $a_{h}$ is a derivation on the algebraic space, it is clear that if $F \mapsto I_{m}^{S}(F)$ is injective, the proposition is proved.

We suppose that for $F \in \mathrm{CO}_{\infty_{-}}$, we have $I_{m}^{S}(F)=0$ and for $z \in \mathbb{C}$, we put $\phi(z)=I_{m}^{S}(z . F)$. We get the following power series

$$
\begin{aligned}
\phi(z) & =\sum_{n} \sum_{J \subset\{1, \ldots, n\}} \int_{\left(S^{1}\right)^{n}}(-1)^{n-|J|}\left\langle D_{S_{J}}^{(2)} F^{n}\left(s_{1}, \ldots, s_{n}\right), z \cdot B\left(s_{1}\right) \otimes \cdots \otimes z \cdot B\left(s_{n}\right)\right\rangle d s_{1} \cdots d s_{n} \\
& =\sum_{n} z^{n} \sum_{J \subset\{1, \ldots, n\}} \int_{\left(S^{1}\right)^{n}}(-1)^{n-|J|}\left\langle D_{S_{J}}^{(2)} F^{n}\left(s_{1}, \ldots, s_{n}\right), B\left(s_{1}\right) \otimes \cdots \otimes B\left(s_{n}\right)\right\rangle d s_{1} \cdots d s_{n} .
\end{aligned}
$$

Since $I_{m}^{S}(F)=0$, we have $\phi(z)=0$. We deduce that for all $n \geq 0$

$$
\sum_{J \subset\{1, \ldots, n\}} \int_{\left(S^{1}\right)^{n}}(-1)^{n-|J|}\left\langle D_{S_{J}}^{(2)} F^{n}\left(s_{1}, \ldots, s_{n}\right), B\left(s_{1}\right) \otimes \cdots \otimes B\left(s_{n}\right)\right\rangle d s_{1} \cdots d s_{n}=0
$$

for all $h \in \mathcal{H}$. It can be written that

$$
\sum_{J \subset\{1, \ldots, n\}} \int_{\left(S^{1}\right)^{n}}(-1)^{n-|J|}\left\langle D_{S_{J}}^{(2)} F^{n}\left(s_{1}, \ldots, s_{n}\right), h\left(s_{1}\right) \otimes \cdots \otimes h\left(s_{n}\right)\right\rangle d s_{1} \cdots d s_{n}=0 .
$$

We use Meyer's isomorphism from $\mathcal{C}^{\infty}\left(S^{1}, \mathbb{R}^{d}\right)$ into $\mathcal{C}^{\infty}\left([0,1], \mathbb{R}^{d}\right)$ given by

$$
\gamma:=\sum_{i, k} a_{i, k} \gamma_{i, k} \mapsto \sum_{i} \sum_{k<0} a_{i, k} \alpha_{k} \gamma_{i, k}+\sum_{i} a_{i, o} \tilde{\gamma}_{i, 0}+\sum_{i} \sum_{k \geq 0} a_{i, k+1} \tilde{\alpha}_{k} \gamma_{i, k}
$$

The coefficients $a_{i, k}$ are for fast diminution, $\tilde{\gamma}_{i, 0}(s)=s . e_{i}$ with $\left|\alpha_{k}\right|<\infty$ for $k>0$ and $\left|\tilde{\alpha}_{k}\right|<\infty$ for $k<0$. For all $\gamma$ we have $\int_{0}^{1}|\gamma(s)|^{2} d s<\infty$ with $\gamma(0)=0$. We deduce

$$
\int_{0<s_{1}<\cdots<s_{n}<1} F\left(s_{1}, \ldots, s_{n}\right) h\left(s_{1}\right) \cdots h\left(s_{n}\right) d s_{1} \cdots d s_{n}=0
$$

for all $h \in \mathcal{C}^{\infty}\left(S^{1}, \mathbb{R}^{d}\right)$, where $F$ is smooth symmetric. $F$ is a smooth symmetric function

$$
F\left(s_{1}, \ldots, s_{n}\right)=\sum_{i_{1}, \ldots, i_{d} \geq 1} F^{i_{1} \cdots i_{d}}\left(s_{1}, \ldots, s_{n}\right) e_{i_{1}} \otimes \cdots \otimes e_{i_{d}}
$$

Let us suppose that $F \neq 0$. Without restriction we can suppose that there exists an $\epsilon>0$, there exist $s_{1}^{(\varepsilon)}<\cdots<s_{d}^{(\varepsilon)}$ and $i_{1}, \ldots, i_{d}$ such that

$$
F^{i_{1} \cdots i_{d}}\left(s_{1}^{(\varepsilon)}, \ldots, s_{d}^{(\varepsilon)}\right)>\varepsilon .
$$

Since $F$ is smooth, there is very small $\eta>0$ such that

$$
F^{i_{1} \cdots i_{d}}\left(s_{1}^{(\varepsilon)}, \ldots, s_{n}^{(\varepsilon)}\right)>\frac{\varepsilon}{2}
$$

on the product $\left[s_{1}^{(\varepsilon)}-\eta, s_{1}^{(\varepsilon)}\right] \times \cdots \times\left[s_{d}^{(\varepsilon)}-\eta, s_{d}^{(\varepsilon)}\right]=\prod I_{k}$. Then we take set $h=\sum 1_{I_{k}} e_{i_{k}}$ which give a contradiction. This shows if $I_{m}^{S}(F)=0$, we have necessary $F=0$. Thus, the map $F \mapsto I_{m}^{S}(F)$ is injective and the proposition is proved. 


\section{Poisson space}

The theory of deformation quantization was initiated in [1, 2]. See [7, 18, 26] for reviews and [9] for basical background. This section gives some properties of the Poisson structure of the Connes space $\mathrm{CO}_{\infty-}$. We make also the quantization deformation of that Poisson structure in Moyal star-product. We note by $\mathbb{K}=\mathbb{R}$ or $\mathbb{C}$.

Definition 5. A Poisson structure on $\mathrm{CO}_{\infty_{-}}$is given by a $\mathbb{K}$-bilinear map $\{\cdot, \cdot\}$ from $\mathrm{CO}_{\infty_{-}-} \times$ $\mathrm{CO}_{\infty-}$ into $\mathrm{CO}_{\infty-}$ such that:

1. $\{\cdot, \cdot\}$ is antisymmetric, satisfies the Jacobi identity and verifies the Leibniz rule for the Wick product of $\mathrm{CO}_{\infty-}$.

2. For all $k, C$, there exists $K, k_{1}, C_{1}$ such that for all $F_{1}, F_{2} \in \mathrm{CO}_{\infty}$ - we get

$$
\left\|\left\{F_{1}, F_{2}\right\}\right\|_{k, C} \leq K\left\|F_{1}\right\|_{k_{1}, C_{1}}\left\|F_{2}\right\|_{k_{1}, C_{1}} .
$$

We note by $\mathrm{CO}_{\infty-}[[\hbar]]$ the set of formal series with coefficients in the Connes space $\mathrm{CO}_{\infty_{-}}$.

Definition 6. A star-product on $\mathrm{CO}_{\infty-}[[\hbar]]$ is a $\mathbb{K}[[h]]$-bilinear map $\star_{\hbar}$ on $\mathrm{CO}_{\infty-}[[\hbar]] \times \mathrm{CO}_{\infty-}[[\hbar]]$ valued in $\mathrm{CO}_{\infty-}[[\hbar]]$. For all $F_{1}, F_{2} \in \mathrm{CO}_{\infty-}$ we have

$$
F_{1} \star_{\hbar} F_{2}=\sum_{r \geq 0} \frac{\hbar^{r}}{r !} P^{r}\left(F_{1}, F_{2}\right) .
$$

For all $r \geq 0, P^{r}$ is a bilinear map on $\mathrm{CO}_{\infty-}$ satisfying:

1. $P^{0}\left(F_{1}, F_{2}\right)=: F_{1} \cdot F_{2}:$.

2. $P^{1}\left(F_{1}, F_{2}\right)-P^{1}\left(F_{2}, F_{1}\right)=2\left\{F_{1}, F_{2}\right\}$.

3. For all $r>0$, for all $k, C>0$ there are $K, k_{1}, C_{1}>0$ such that for all $F_{1}, F_{2} \in \mathrm{CO}_{\infty-}$, we get

$$
\left\|P^{r}\left(F_{1}, F_{2}\right)\right\|_{k, C} \leq K\left\|F_{1}\right\|_{k_{1}, C_{1}}\left\|F_{2}\right\|_{k_{1}, C_{1}}
$$

4. For all $F_{1}, F_{2}, F_{3} \in \mathrm{CO}_{\infty-}$, we have: $F_{1} \star_{\hbar}\left(F_{2} \star_{\hbar} F_{3}\right)=\left(F_{1} \star_{\hbar} F_{2}\right) \star_{\hbar} F_{3}$.

We call $\star_{\hbar}$ a deformation of the Poisson bracket on $\mathrm{CO}_{\infty-}$.

Definition 7. Two deformation quantizations $\star_{\hbar}^{1}$ and $\star_{\hbar}^{2}$ of the same Poisson bracket are said equivalent if there exists a $\mathbb{K}[[\hbar]]$-linear map $T: \mathrm{CO}_{\infty_{-}}[[\hbar]] \rightarrow \mathrm{CO}_{\infty_{-}}[[\hbar]]$ expressed as a formal $T=I+\sum_{r \geq 1} \hbar^{r} T_{r}$ satisfying:

1. For all $r \geq 1, T_{r}: \mathrm{CO}_{\infty-} \rightarrow \mathrm{CO}_{\infty-}$ is a continuous operators and $T_{0}$ is the identity operator of $\mathrm{CO}_{\infty-}$.

2. For all $F, G \in \mathrm{CO}_{\infty-}$, we have $T\left(F \star_{\hbar}^{1} G\right)=T(F) \star_{\hbar}^{2} T(G)$.

Let us consider $\omega=\left(\omega_{i j}\right)_{i, j \geq 1}$ a non-degenerate bilinear and antisymmetric form in $\mathbb{R}^{d} \oplus \mathbb{R}^{d}$. We define the bilinear and antisymmetric form $\Omega$ on $\mathcal{H}\left(S^{1}, \mathbb{R}^{d}\right)$. For all $\gamma_{1}, \gamma_{2} \in \mathcal{H}$

$$
\Omega\left(\gamma_{1}, \gamma_{2}\right)=\int_{S^{1}} \omega\left(\gamma_{1}(s), \gamma_{2}(s)\right) d s
$$

We recall that $\gamma_{i, k}$ are given by (3) and (4). We have

$$
\Omega\left(\gamma_{i, k_{i}}, \gamma_{j, k_{j}}\right)=\frac{\omega_{i j}}{C k^{2}+1} \delta_{k_{i} k_{j}},
$$


where $\delta_{k_{i} k_{j}}$ is the Kronecker delta. We note by $\omega_{i j}=\left(\omega^{i j}\right)^{-1}$. The Poisson matrix of $\Omega$ is given by

$$
\left\{\gamma_{i, k_{i}}, \gamma_{j, k_{j}}\right\}=\left(C k^{2}+1\right) \cdot \omega^{i j} \cdot \delta_{k_{i} k_{j}} .
$$

Let us note by $a_{\gamma_{i, k}}$ the annihilation operator associated to $\gamma_{i, k}$. Then, for all $F, G \in \mathrm{CO}_{\infty-}$ we have

$$
\{F, G\}=\sum_{m, n} \sum_{i, j, k}\left(C k^{2}+1\right) \omega^{i j} \times: a_{\gamma_{i, k}} F^{n} \cdot a_{\gamma_{j, k}} G^{m}: .
$$

Proposition 3. $\{\cdot, \cdot\}$ defines a Poisson structure on $\mathrm{CO}_{\infty}$ in the sense of Definition 5 .

Proof. Since the Wick product is continuous, for all $k, C>0$, there are $k_{2}, C_{2}>0$ such that

$$
\|\{F, G\}\|_{k, C} \leq \sum_{m, n, k}\left(C k^{2}+1\right)\left\|a_{\gamma_{i, k}} F^{n}\right\|_{k_{2}, C_{2}}\left\|a_{\gamma_{j, k}} G^{m}\right\|_{k_{2}, C_{2}} .
$$

Using integration by parts, there are $k_{3}, C_{3}>0$ large enough such that

$$
\|\{F, G\}\|_{k, C} \leq \sum_{m, n, k}\left(C k^{2}+1\right)^{-1}\left\|F^{n}\right\|_{k_{3}, C_{3}}\left\|G^{m}\right\|_{k_{3}, C_{3}} .
$$

Then

$$
\|\{F, G\}\|_{k, C} \leq\left(\sum\left\|F^{n}\right\|_{k_{3}, C_{3}}\right)\left(\sum\left\|G^{n}\right\|_{k_{3}, C_{3}}\right)\left(\sum \frac{1}{\left(C k^{2}+1\right)}\right) .
$$

Finally

$$
\|\{F, G\}\|_{k, C} \leq K\|F\|_{k_{3}, C_{3}}\|G\|_{k_{3}, C_{3}}<\infty .
$$

Remark 6. Integration by parts allows us to change the factor $\left(C k^{2}+1\right)$ into $\left(C k^{2}+1\right)^{-1}$ in the proof and give a bounded form of the Poisson bracket. Thus, $\Omega$ acts continuously on the space $\mathrm{CO}_{\infty-}$.

Using the Wick product, we can define the powers of the Poisson bracket as following: for all $r \geq 0$ and $F, G \in \mathrm{CO}_{\infty-}$

$$
P^{r}(F, G)=\sum_{\substack{n, m \\ i_{1}, \ldots, i_{r} \geq 1 \\ j_{1}, \ldots, j_{r} \geq 1 \\ k_{1}, \ldots, k_{r} \geq 1}} \prod_{j=1}^{r}\left(C k_{j}^{2}+1\right) \omega^{i_{1} j_{1}} \cdots \omega^{i_{r} j_{r}}: a_{\gamma_{i_{1}, k_{1}}} \cdots a_{\gamma_{i_{r}, k_{r}}} F^{n} \cdot a_{\gamma_{j_{1}, k_{1}}} \cdots a_{\gamma_{j_{r}, k_{r}}} G^{m} .
$$

Then we have

Definition 8. The Moyal star-product on $\mathrm{CO}_{\infty-}$ is given by

$$
F \star_{\hbar} G=: F . G:+\sum_{r \geq 1} \frac{\hbar^{r}}{r !} P^{r}(F, G) .
$$

The Moyal star-product endowed with the symplectic structure of $\Omega$ is well defined on $\mathrm{CO}_{\infty-}$ in the sense of Definition 6. Then, we have

Theorem 3. The formula (12) defines a deformation quantization of $\{\cdot, \cdot\}$ on $\mathrm{CO}_{\infty-}$ in the framework of Definition 6. 
Proof. Since the Wick product is continuous, for all $k, C>0$ there are $k_{1}^{\prime}, C_{1}^{\prime}>0$ such that

$$
\left\|P^{r}(F, G)\right\|_{k, C} \leq \sum_{m, n, k} \prod_{j}\left(C k_{j}^{2}+1\right)\left\|a_{\gamma_{i_{1}, k_{1}}} \cdots a_{\gamma_{i_{r}, k_{r}}} F^{n}\right\|_{k_{1}^{\prime}, C_{1}^{\prime}}|| a_{\gamma_{j_{1}, k_{1}}} \cdots a_{\gamma_{j_{r}, k_{r}}} G^{m} \|_{k_{1}^{\prime}, C_{1}^{\prime}} .
$$

Using integration by parts, there are $k_{2}^{\prime}, C_{2}^{\prime}>0$ large enough such that

$$
\left\|P^{r}(F, G)\right\|_{k, C} \leq \sum \prod_{j}^{r}\left(C k_{j}^{2}+1\right)^{-1}\left\|F^{n}\right\|_{k_{2}^{\prime}, C_{2}^{\prime}}\left\|G^{m}\right\|_{k_{2}^{\prime}, C_{2}^{\prime}} .
$$

Without loss of generality, we get

$$
\left\|P^{r}(F, G)\right\|_{k, C} \leq\left(\sum\left\|F^{n}\right\|_{k_{2}^{\prime}, C_{2}^{\prime}}\right)\left(\sum\left\|G^{m}\right\|_{k_{2}^{\prime}, C_{2}^{\prime}}\right)\left(\sum \prod_{j}^{r} \frac{1}{\left(C k_{j}^{2}+1\right)}\right) .
$$

Then

$$
\left\|P^{r}(F, G)\right\|_{k, C} \leq K^{\prime}\|F\|_{k_{2}^{\prime}, C_{2}^{\prime}}\|G\|_{k_{2}^{\prime}, C_{2}^{\prime}}<\infty .
$$

It remains to check the algebraic properties. It is enough to prove them if we consider finite sum of $\gamma_{N}$ because $P^{r}$ apply the product of this space on itself and because by Stone-Weierstrass theorem the set of finite sum of $\gamma_{N}$ is dense in $C 0_{\infty-}$. But in [15], these algebraic properties were proved where a completion of Hida type of the set of finite sum (by using an Hida Fock space) was chosen.

The result holds.

Remark 7. Since the map $F \mapsto I_{m}^{S}(F)$ is injective, we can use the dictionary between the multiple Stratonovich integrals and the algebraic model $\mathrm{CO}_{\infty_{-}}$. Then, the formula (12) becomes

$$
I_{m}^{S}(F) \star_{\hbar} I_{m}^{S}(G)=I_{m}^{S}(F) \cdot I_{m}^{S}(G)+\sum_{r \geq 1} \frac{\hbar^{r}}{r !} P^{r}\left(I_{m}^{S}(F), I_{m}^{S}(G)\right) .
$$

On $I_{m}^{S} F$ we choose the Banach norm of $F$.

\section{Equivalence of deformation quantization}

Using the model of [3], we show that there are many similarities between the Connes space we use here and the Hida test algebra of [15]. Let us consider the Hilbert space $\mathcal{H}=\mathcal{H}\left(S^{1}, \mathbb{R}^{d}\right)$ of functions $\gamma$ defined from the circle into $\mathbb{R}^{d}$ such that

$$
\|\gamma\|^{2}=\int_{0}^{1}|\gamma(s)|^{2} d s+\int_{0}^{1}\left|\frac{d}{d s} \gamma(s)\right|^{2} d s<\infty .
$$

Let $\left(e_{i}\right)_{1 \leq i \leq d}$ be the canonical basis of $\mathbb{R}^{d}$. Considering the Fourier basis of $\mathcal{H}$ defined by (3) and (4), we can define on the Hilbert space $\mathcal{H} \oplus \mathcal{H}^{*}$ a Poisson structure by $\Omega\left(\Gamma_{1}, \Gamma_{2}\right)=$ $\int_{0}^{1} \omega\left(\Gamma_{1}(s), \Gamma_{2}(s)\right) d s+\int_{0}^{1} \omega\left(\frac{d}{d s} \Gamma_{1}(s), \frac{d}{d s} \Gamma_{2}(s)\right) d s$, where $\omega=\left(\omega_{i j}\right)_{i, j \geq 1}$ is a non-degenerated bilinear and antisymmetric form of $\mathbb{R}^{d} \oplus \mathbb{R}^{d}$ such that for all $i \neq j$

$$
\omega_{i j}=0, \quad \omega_{i i^{*}}=1, \quad \omega_{i^{*} j^{*}}=0, \quad \omega_{i^{*} i}=-1,
$$

and note $\Gamma_{j \in\{1,2\}}=\gamma_{j} \oplus \gamma_{j}^{*} \in \mathcal{H} \oplus \mathcal{H}^{*}$. We get that $\Omega$ acts continuously on $\mathrm{CO}_{\infty-} \times \mathrm{CO}_{\infty_{-}}$and its Poisson matrix is bounded. Then, the model of [3] holds for the rest of the section. 
Definition 9. For all $\gamma \oplus \gamma^{*} \in \mathcal{H} \oplus \mathcal{H}^{*}$, we call Wick exponentials, the maps $\Phi_{\gamma, \gamma^{*}}$ defined by

$$
h \oplus h^{*} \in \mathcal{H} \oplus \mathcal{H}^{*} \mapsto \Phi_{\gamma, \gamma^{*}}\left(h, h^{*}\right):=\exp \left(\langle h, \gamma\rangle+\left\langle h^{*}, \gamma^{*}\right\rangle\right) .
$$

We get a classical result for Hida calculus given by

Proposition 4. The Wick exponentials are dense in $\mathrm{CO}_{\infty_{-}}$.

Proof. We shall note by $\mathrm{CO}_{k, C}^{W}$ the adherence of Wick exponentials in $\mathrm{CO}_{k, C}$ and by $\mathrm{CO}_{k, C}^{n}$ the space of the product of $n$ homogeneous polynomials of $\mathrm{CO}_{k, C}$. We are going to use recurrence on the holomorphic function

$$
F(\lambda)=\exp \left[\lambda\left(\int_{S^{1}}\langle\Gamma(s), T(s)\rangle d s+\int_{S^{1}}\left\langle\frac{d}{d s} \Gamma(s), d T(s)\right\rangle\right)\right],
$$

where $\Gamma=\gamma \oplus \gamma^{*}$ and $T=B \oplus B^{*}$. Then, $F(\lambda)$ can be written under the form

$$
F(\lambda)=\sum_{n \geq 0} \frac{\lambda^{n}}{n !}\left(\int_{S^{1}}\langle\Gamma(s), T(s)\rangle d s+\int_{S^{1}}\left\langle\frac{d}{d s} \Gamma(s), d T(s)\right\rangle\right)^{n},
$$

and obviously $F(\lambda) \in \mathrm{CO}_{k, C}^{W}$. With Cauchy's differentiation formula

$$
F^{(n)}(\lambda)=\frac{n !}{2 i \pi} \int_{S^{1}} \frac{F(z)}{(z-\lambda)^{n+1}} d z .
$$

It is clear that for all $n \geq 0$

$$
\left(\int_{S^{1}}\langle\Gamma(s), T(s)\rangle d s+\int_{S^{1}}\left\langle\frac{d}{d s} \Gamma(s), d T(s)\right\rangle\right)^{n} \in \mathrm{CO}_{k, C}^{W} .
$$

Now, it remains just to prove that all products of $n$ homogeneous polynomials are in the adherence. We consider for $|z|<1$ the holomorphic function

$$
F_{n+1}(z)=\int_{S^{1}}\left\langle\Gamma(s)+z \cdot \Gamma_{1}(s), T(s)\right\rangle d s+\int_{S^{1}}\left(\left\langle\frac{d}{d s} \Gamma(s)+z \cdot \frac{d}{d s} \Gamma_{1}(s), d T(s)\right\rangle\right)^{n+1} .
$$

$F_{n+1}$ is clearly in $\mathrm{CO}_{k, C}^{W}$ and by Cauchy's differentiation formula

$$
F_{n+1}^{\prime}(z)=\frac{1}{2 i \pi} \int_{S^{1}} \frac{F(u)}{(u-z)^{2}} d u .
$$

Then $F_{n+1}^{\prime}$ is also a function of $\mathrm{CO}_{k, C}^{W}$. By computation, we get

$$
F_{n+1}^{\prime}(z)=(n+1)\left(\int_{S^{1}}\left\langle\Gamma_{1}(s), T(s)\right\rangle d s+\int_{S^{1}} \frac{d}{d s}\left\langle\Gamma_{1}(s), d T(s)\right\rangle\right) \cdot F_{n}(z) .
$$

Then

$$
\begin{aligned}
F_{n+1}^{\prime}(0)= & (n+1)\left(\int_{S^{1}}\left\langle\Gamma_{1}(s), T(s)\right\rangle d s+\int_{S^{1}} \frac{d}{d s}\left\langle\Gamma_{1}(s), d T(s)\right\rangle\right) \\
& \times\left(\int_{S^{1}}\langle\Gamma(s), T(s)\rangle d s+\int_{S^{1}}\left\langle\frac{d}{d s} \Gamma(s), d T(s)\right\rangle\right)^{n} .
\end{aligned}
$$

Thus, we proved the recurrence relation in the order $(n+1)$. We have

$$
\sum \prod_{i=1}^{n} \int_{S^{1}}\left\langle\Gamma_{i}(s), T(s)\right\rangle d s+\int_{S^{1}}\left\langle\frac{d}{d s} \Gamma_{i}(s), d T\right\rangle \in \mathrm{CO}_{k, C}^{W} .
$$


By the theorem of Stone-Weierstrass, for all $k, C>0$ we get that

$$
I_{m}^{S}(F) \in \mathrm{CO}_{k, C}^{W}
$$

because $F$ is a limit of elements of $\mathrm{CO}_{k, C}^{n}$. We conclude that

$$
\bigcap_{k, C} \mathrm{CO}_{k, C}^{W}=\mathrm{CO}_{\infty-}^{W}=\mathrm{CO}_{\infty-}
$$

The proposition is proved.

According to [15], we choose the operator $A: \gamma_{i, k} \mapsto \alpha_{k} \gamma_{i, k}$ such that $\left|\alpha_{k}\right| \leq K|k|^{\mu}$ for some $\mu>0$. We put

$$
E_{A}\left(I_{m}^{S}(F), I_{m}^{S}(G)\right)=\sum_{i, k \geq 1}\left[\alpha_{k} D_{\gamma_{i, k}} I_{m}^{S}(F) D_{\gamma_{i, k}^{*}} I_{m}^{S}(G)+\alpha_{k} D_{\gamma_{i, k}} I_{m}^{S}(G) D_{\gamma_{i, k}^{*}} I_{m}^{S}(F)\right]
$$

where $D_{\gamma_{i, k}}$ (resp. $D_{\gamma_{i, k}^{*}}$ ) is the Gâteaux derivative at $\gamma_{i, k}\left(\right.$ resp. $\left.\gamma_{i, k}^{*}\right)$ in the direction $\mathcal{H}$ (resp. $\left.\mathcal{H}^{*} \sim \mathcal{H}\right)$.

Theorem 4. $E_{A}$ is continuous from $\mathrm{CO}_{\infty-} \times \mathrm{CO}_{\infty-}$ into $\mathrm{CO}_{\infty-}$.

Proof. For all $k, C>0$, integrating by parts we can find $k_{2}, C_{2}>0$ large enough such that

$$
\left\|E_{A}\left(I_{m}^{S}(F), I_{m}^{S}(G)\right)\right\|_{k, C} \leq \sum\left(C k^{2}+1\right)^{-1}\left\|I_{m}^{S}(F)\right\|_{k_{2}, C_{2}}\left\|I_{m}^{S}(G)\right\|_{k_{2}, C_{2}}<\infty .
$$

This proves the theorem.

We put

$$
C_{1}^{A}\left(I_{m}^{S}(F), I_{m}^{S}(G)\right)=\left\{I_{m}^{S}(F), I_{m}^{S}(G)\right\}+E_{A}\left(I_{m}^{S}(F), I_{m}^{S}(G)\right) .
$$

Using (11), we get

$$
\begin{aligned}
C_{1}^{A}\left(I_{m}^{S}(F), I_{m}^{S}(G)\right)= & \sum_{i, k \geq 1}\left[\left(\alpha_{k}+1\right) D_{\gamma_{i, k}} I_{m}^{S}(F) D_{\gamma_{i, k}^{*}} I_{m}^{S}(G)\right. \\
& \left.+\left(\alpha_{k}-1\right) D_{\gamma_{i, k}} I_{m}^{S}(G) D_{\gamma_{i, k}^{*}} I_{m}^{S}(F)\right] .
\end{aligned}
$$

Then, we put in the sense of differential operators

$$
C_{r}^{A}\left(I_{m}^{S}(F), I_{m}^{S}(G)\right)=\left(C_{1}^{A}\right)^{r}\left(I_{m}^{S}(F), I_{m}^{S}(G)\right) .
$$

We get

$$
\begin{aligned}
C_{r}^{A}\left(I_{m}^{S}(F), I_{m}^{S}(G)\right)= & \sum_{\substack{i_{1}, \ldots, i_{r} \geq 1 \\
k_{1}, \ldots, k_{r} \geq 1}} \prod_{l=1}^{r}\left(\alpha_{k_{l}}+1\right) D_{\gamma_{i_{1}, k_{1}}^{\sharp}} \cdots D_{\gamma_{i_{r}, k_{r}}^{\sharp}} I_{m}^{S}(F) \\
& \times\left(\alpha_{k_{l}}-1\right) D_{\gamma_{i_{1}, k_{1}}^{\sharp}} \cdots D_{\gamma_{i_{r}, k_{r}}^{\sharp}} I_{m}^{S}(G) .
\end{aligned}
$$

We note by $\gamma_{i, k}^{\sharp}=\gamma_{i, k}$ or $\gamma_{i, k}^{*}$ to avoid additional terms due to the symmetry. Clearly, $C_{r}^{A}$ is continuous from $\mathrm{CO}_{\infty_{-}} \times \mathrm{CO}_{\infty_{-}}$into $\mathrm{CO}_{\infty_{-}-}$. We can find $k_{0}, C_{0}>0$ large enough and $K>0$, by referring to the proof of Theorem 4 , such that

$$
\left\|C_{r}^{A}\left(I_{m}^{S}(F), I_{m}^{S}(G)\right)\right\|_{k, C} \leq K\left\|I_{m}^{S}(F)\right\|_{k_{0}, C_{0}}\left\|I_{m}^{S}(G)\right\|_{k_{0}, C_{0}}<\infty
$$


Definition 10. We put

$$
I_{m}^{S}(F) \star_{\hbar}^{A} I_{m}^{S}(G)=I_{m}^{S}(F) \cdot I_{m}^{S}(G)+\sum_{r \geq 1} \frac{\hbar^{r}}{r !} C_{r}^{A}\left(I_{m}^{S}(F), I_{m}^{S}(G)\right) .
$$

(13) defines a deformation quantization of $\{\cdot, \cdot\}$ in the sense of Definition 6.

Finally, we have

Proposition 5. $\star_{\hbar}^{A}$ and $\star_{\hbar}$ are equivalent on the Connes space $\mathrm{CO}_{\infty-}$.

Proof. We put as in [3], for all $I_{m}^{S}(F) \in \mathrm{CO}_{\infty-}$

$$
T_{1} I_{m}^{S}(F)=-\sum_{i, k \geq 1} \alpha_{k} D_{\gamma_{i, k}} D_{\gamma_{i, k}^{*}} I_{m}^{S}(F)
$$

Then, integrating by parts $\forall k, C>0$, there exist $k_{1}>0$ and $C_{1}>0$ large enough such that

$$
\left\|T_{1} I_{m}^{S}(F)\right\|_{k, C} \leq \sum\left(C k^{2}+1\right)^{-1}\left\|I_{m}^{S}(F)\right\|_{k_{1}, C_{1}}<\infty
$$

So $T_{1}$ is continuous on $\mathrm{CO}_{\infty-}$. We put $T:=\exp \left(\hbar T_{1}\right)$. $T$ is the formal series of operators

$$
T I_{m}^{S}(F)=\sum_{r \geq 0} \frac{(-\hbar)^{r}}{r !} \sum_{\substack{i_{1}, \ldots, i_{r} \geq 1 \\ k_{1}, \ldots, k_{r} \geq 1}} \prod_{l=1}^{r} \alpha_{k_{l}} D_{\gamma_{i_{1}, k_{1}}} \cdots D_{\gamma_{i_{r}, k_{r}}} D_{\gamma_{i_{1}, k_{1}}^{*}} \cdots D_{\gamma_{i_{r}, k_{r}}^{*}} I_{m}^{S}(F)
$$

where we have seen that

$$
T^{r}\left(I_{m}^{S}(F)\right):=\sum_{\substack{i_{1}, \ldots, i_{r} \geq 1 \\ k_{1}, \ldots, k_{r} \geq 1}} \prod_{l=1}^{r} \alpha_{k_{l}} D_{\gamma_{i_{1}, k_{1}}} \cdots D_{\gamma_{i_{r}, k_{r}}} D_{\gamma_{i_{1}, k_{1}}^{*}} \cdots D_{\gamma_{i_{r}, k_{r}}^{*}} I_{m}^{S}(F) .
$$

Since $T^{r}=\underbrace{T_{1} \circ \cdots \circ T_{1}}_{r \text {-times }}$, we get that $T^{r}$ is continuous and as a result $T$ is continuous.

We note by $\langle\cdot, \cdot\rangle_{c}: \mathcal{H} \times \mathcal{H}^{*} \rightarrow \mathbb{R}$ the canonical pairing between $\mathcal{H}$ and $\mathcal{H}^{*}$. Then, according to [3], we have the formula

$$
\Phi_{\gamma_{1}, \gamma_{1}^{*}} \star_{\hbar}^{A} \Phi_{\gamma_{2}, \gamma_{2}^{*}}=\exp \left[\hbar\left(\left\langle(A+I) \gamma_{1}, \gamma_{2}^{*}\right\rangle_{c}+\left\langle(A-I) \gamma_{2}, \gamma_{1}^{*}\right\rangle_{c}\right)\right] \Phi_{\gamma_{1}+\gamma_{2}, \gamma_{1}^{*}+\gamma_{2}^{*}} .
$$

Then as in [3], we get

$$
T\left(\Phi_{\gamma_{1}, \gamma_{1}^{*}} \star_{\hbar}^{A} \Phi_{\gamma_{2}, \gamma_{2}^{*}}\right)=T\left(\Phi_{\gamma_{1}, \gamma_{1}^{*}}\right) \star_{\hbar} T\left(\Phi_{\gamma_{2}, \gamma_{2}^{*}}\right) .
$$

This proves the proposition since the Wick exponentials are dense in the Connes space $\mathrm{CO}_{\infty-}$.

Remark 8. In the Connes space $\mathrm{CO}_{\infty-}$ endowed with Stratonovich chaos, unlike in [3], Moyal star-product and normal star-product $(A=I)$ are obviously equivalent. We can suppose that equivalences of [15] with the Hida test functional space remain true because the Connes space $\mathrm{CO}_{\infty-}$ is very small. 


\section{References}

[1] Bayen F., Flato M., Fronsdal C., Lichnerowicz A., Sternheimer D., Deformation theory and quantization. I. Deformations of symplectic structures, Ann. Physics 111 (1978), 61-110.

[2] Bayen F., Flato M., Fronsdal C., Lichnerowicz A., Sternheimer D., Deformation theory and quantization. II. Physical applications, Ann. Physics 111 (1978), 111-151.

[3] Dito G., Deformation quantization on a Hilbert space, in Noncommutative Geometry and Physics, World Sci. Publ., Hackensack, NJ, 2005, 139-157, math.QA/0406583.

[4] Dito J., Star-products and nonstandard quantization for Klein-Gordon equation, J. Math. Phys. 33 (1992), 791-801.

[5] Dito J., Star-product approach to quantum field theory: the free scalar field, Lett. Math. Phys. 20 (1990), $125-134$.

[6] Dito G., Léandre R., Stochastic Moyal product on the Wiener space, J. Math. Phys. 48 (2007), 023509, 8 pages.

[7] Dito G., Sternheimer D., Deformation quantization: genesis, developments and metamorphoses, in Deformation Quantization (Strasbourg, 2001), IRMA Lect. Math. Theor. Phys., Vol. 1, 9-54, math.QA/0201168.

[8] Dütsch M., Fredenhagen K., Perturbative algebraic field theory, and deformation quantization, in Mathematical Physics in Mathematics and Physics (Siena, 2000), Fields Inst. Commun., Vol. 30, Amer. Math. Soc., Providence, RI, 2001, 151-160.

[9] Gerstenhaber M., On the deformation of rings and algebras, Ann. of Math. (2) 79 (1964), 59-103.

[10] Getzler E., Cyclic cohomology and the path integral of Dirac operator, unpublished.

[11] Hida T., Kuo H.H., Potthoff J., Streit L., White noise, Mathematics and its Applications, Vol. 253, Kluwer Academic Publishers Group, Dordrecht, 1993.

[12] Hu Y.Z., Meyer P.A., Sur les intégrales multiples de Stratonovitch, in Séminaire de Probabilités, XXII, Lecture Notes in Math., Vol. 1321, Springer, Berlin, 1988, 72-81.

[13] Léandre R., Connes-Hida calculus in index theory, in XIVth International Congress on Mathematical Physics, World Sci. Publ., Hackensack, NJ, 2005, 493-497.

[14] Léandre R., Deformation quantization in infinite dimensional analysis, in Trends in Stochastic Analysis, London Math. Soc. Lecture Note Ser., Vol. 353, Cambridge Univ. Press, Cambridge, 2009, 303-325.

[15] Léandre R., Deformation quantization in white noise analysis, SIGMA 3 (2007), 027, 8 pages, math.QA/0702624.

[16] Léandre R., Fedosov quantization in white noise analysis, J. Nonlinear Math. Phys. 15 (2008), suppl. 3, 251-263.

[17] Léandre R., Wiener analysis and cyclic homology, in Stochastic Analysis and Mathematical Physics (SAMP/ANESTOC 2002), World Sci. Publ., River Edge, NJ, 2004, 115-127.

[18] Maeda Y., Deformation quantization and noncommutative differential geometry, Sugaku Expositions 16 (2003), 1-23.

[19] Malliavin P., Stochastic analysis, Grundlehren der Mathematischen Wissenschaften, Vol. 313, SpringerVerlag, Berlin, 1997.

[20] Malliavin P., Stochastic calculus of variation and hypoelliptic operators, in Proceedings of the International Symposium on Stochastic Differential Equations (Res. Inst. Math. Sci., Kyoto Univ., Kyoto, 1976), Wiley, New York, 1978, 195-263.

[21] Meyer P.A., Quantum probability for probabilists, Lecture Notes in Mathematics, Vol. 1538, Springer-Verlag, Berlin, 1993.

[22] Nualart D., The Malliavin calculus and related topics, Probability and its Applications (New York), SpringerVerlag, New York, 1995.

[23] Obata N., White noise calculus and Fock space, Lecture Notes in Mathematics, Vol. 1577, Springer-Verlag, Berlin, 1994.

[24] Solé J.L., Utzet F., Intégrale multiple de Stratonovich pour le processus de Poisson, in Séminaire de Probabilités, XXV, Lecture Notes in Math., Vol. 1485, Springer, Berlin, 1991, 270-283.

[25] Üstünel A.S., An introduction to analysis on Wiener space, Lecture Notes in Mathematics, Vol. 1610, Springer-Verlag, Berlin, 1995.

[26] Weinstein A., Deformation quantization, Astérisque (1995), no. 227, Exp. No. 789, 5, 389-409.

[27] Witten E., Noncommutative geometry and string field theory, Nuclear Phys. B 268 (1986), 253-294. 